\title{
Suppression of Human GD3 Synthase (hST8Sia I) Expression Induced by Retinoic Acid in Human Melanoma SK-MEL-2 Cells
}

\author{
Haw-Young Kwon, Nam-Young Kang and Young-Choon Lee* \\ Department of Biotechnology, College of Natural Resources and Life Science, Dong-A University, Busan 604-714, Korea
}

Received February 8, 2010 /Accepted March 13, 2010

\begin{abstract}
To elucidate the mechanism underlying the suppressive regulation of hST8Sia I expression in retinoic acid (RA)-induced SK-MEL-2 cells, we characterized the promoter region of the hST8Sia I gene. Functional analysis of the 5 -flanking region of the hST8Sia I gene by the transient expression method showed that the -1146 to -646 region, which contains putative binding sites for transcription factors c-Ets-1, CREB, AP-1 and NF- $\mathrm{B}$, functions as the RA-repressive promoter in SK-MEL-2 cells. Site-directed mutagenesis and ChIP analyses indicated that the NF-кB binding site at -731 to -722 is crucial for the RA-induced repression of hST8Sia I in SK-MEL-2 cells. In addition, the transcriptional activity of hST8Sia I suppressed by RA in SK-MEL-2 cells was strongly inhibited by extracellular signal-regulated protein kinase (ERK) inhibitor U0126 and protein kinase C (PKC) inhibitor GÖ6976, as determined by RT-PCR and luciferase assay of hST8Sia I promoter containing the -1146 to -646 regions. These results suggest that RA markedly modulates transcriptional regulation of hST8Sia I gene expression through the PKC/ERK signal pathway in SK-MEL-2 cells.
\end{abstract}

Key words : Human GD3 synthase, retinoic acid, transcription factor, SK-MEL-2

\section{Introduction}

Gangliosides, the sialic acid (NeuAc)-containing glycosphingolipids, are component molecules of the outer leaflet of the plasma membrane of vertebrate cells [21]. They are known to play a pivotal role in tumor formation and progression [8] and thus have been studied as molecules as tumor markers of neuroectoderm-derived malignant cells [9], including melanomas [14,16] and neuroblastomas [3].

Ganglioside GD3 is highly expressed in human melanoma tissues and melanoma cell lines [4,17]. Furthermore, GD3 appears in activated human $\mathrm{T}$ lymphocytes [6] as well as in T-cell acute lymphoblastic leukemia cells [13]. In particular, GD3 and GD2 have been considered to be important molecules not only for the tumor markers, but as targets of antibody therapy $[3,7,26]$. GD3 synthase (ST8Sia I, EC 2.4.99.8) is a key enzyme for the synthesis of whole b-series gangliosides including GD2 in addition to GD3 itself [22]. In general GD3 expression appears to be regulated at the transcriptional level of ST8Sia I gene $[10,19]$. To understand transcriptional regulation mechanism for ST8Sia I gene expression in these biologically important events, it is very important to characterize the promoter function of ST8Sia I gene.

*Corresponding author

Tel : +82-51-200-7591, Fax : +82-51-200-6536

E-mail : yclee@dau.ac.kr
Especially, regulatory mechanisms for GD3 expression in human melanoma cells is of quite importance, since GD3 is well known as a human melanoma-specific antigen $[4,24]$. Recently, we have elucidated for the first time transcriptional regulation mechanism of human GD3 synthase (hST8Sia I) expression necessary for GD3 synthesis highly expressed in human melanoma cells [11].

Retinoic acid (RA), a group of natural and synthetic ana$\operatorname{logs}$ of vitamin $\mathrm{A}$, is well known to induce differentiation and inhibit proliferation of a variety of tumor cell lines including melanoma cells in culture $[5,6,20]$. Recently, we firstly observed a dramatic suppression of hST8Sia I mRNA expression in the presence of RA in human melanoma SK-MEL-2 cells. In this study, the promoter region to direct down-regulation of hST8Sia I gene transcription by RA in SK-MEL-2 cells was functionally characterized. The present results clearly indicate that the NF- $\mathrm{BB}$ binding site of the hST8Sia I promoter plays an important role in down-regulation of hST8Sia I gene expression induced by RA in melanoma.

\section{Materials and Methods}

\section{Cell cultures}

The SK-MEL-2 human melanoma cell [11] was grown in Dulbecco's modified Eagle's medium (DMEM) (WelGENE 
Co., Korea) supplemented with $10 \%$ heat-inactivated fetal bovine serum (FBS), $100 \mathrm{U} / \mathrm{ml}$ penicillin, and $100 \mu \mathrm{g} / \mathrm{ml}$ streptomycin at $37^{\circ} \mathrm{C}$ under $5 \% \mathrm{CO}_{2}$.

Reverse transcription-polymerase chain reaction
(RT-PCR)

Total RNA was isolated from SK-MEL-2 cells using Trizol reagent (Invitrogen, USA). Two micrograms of RNA was subjected to reverse transcription with random nonamers using Takara RNA PCR kit (Takara, Japan) according to the manufacturer's protocol. The cDNA was amplified by PCR with the following primers: hST8Sia I (460 bp), 5'TGTGGTCCAGAA AGACATTTGTGGACA-3' (sense) and 5'-TGGAGTGAGGTATCTTCACATGGGTCC-3' (antisense); $\beta$-actin (247 bp), 5'-CAAGAGATGGCCACGGCTGCT-3' (sense) and 5'-TCCTTCTGCATCCTGTCGGCA-3' (antisense). The PCR product for GD3 synthase was subcloned into pGEM-T vector (Promega, USA) and then sequenced. These genes were found to be identical to the expected cDNA.

\section{Preparation of reporter plasmids}

To identify the minimal promoter sequence in the 5 '-flanking region of the hST8Sia I gene, luciferase reporter plasmids [12], Group I; -2000/pGL3, -2646 -646/pGL3, $-2499 \sim-499 /$ pGL3, Group II; -2646 -646/pGL3 and its derivatives $(-1146 \sim-646 /$ pGL3 to $-2246 \sim-646 /$ pGL3) and Group III; -2646 -646/pGL3 and its different derivatives( $-646 \sim-1146 /$ pGL3, $-1146 \sim-1646 /$ pGL3, -1646 -2146/pGL3 and -2146 -2646/pGL3 ), were used. The promoterless and enhancerless luciferase vector pGL3-Basic and the pGL3Control with SV40 promoter and enhancer (Promega, USA) were used as negative and positive controls, respectively. Mutant plasmids [12] with base substitution at the CREB, AP-1, c-Ets-1, NF- $k B$ binding sites were also used to identify the transcription factor-binding sites contributing to transcriptional regulation of hST8Sia I gene in SK-MEL-2 cells.

\section{Transient transfection and reporter assay}

To analyze hST8Sia I promoter activity in response to RA treatment, SK-MEL-2 cells $\left(3.0 \times 10^{5}\right.$ cells/well $)$ were seeded in 24-well tissue culture plates and allowed to grow to $70 \%$ confluence, at which point they were transiently co-transfected with $0.5 \mu \mathrm{g}$ of the indicated reporter plasmid and 50 ng of the control Renilla luciferase vector pRL-TK (Promega, USA), using $1 \mu \mathrm{l}$ Lipofectamine 2000 (Invitrogen, USA). After a $12 \mathrm{hr}$ recovery in normal medium without RA, the medium was changed to medium containing $70 \mu \mathrm{M}$ RA and incubated for an additional $12 \mathrm{hr}$, after which cells were collected and treated with passive lysis buffer (Promega, USA). Firefly and Renilla luciferase activities were measured using the Dual-Luciferase Reporter Assay System (Promega, USA), according to the manufacturer's instructions, and a GloMax $^{\mathrm{TM}}$ 20/20 luminometer (Promega, USA). Firefly luciferase activity of the reporter plasmid was normalized to Renilla luciferase activity and expressed as a fold induction over the empty pGL3-Basic vector, used as a negative control. Independent triplicate experiments were performed for each plasmid.

\section{Chromatin immunoprecipitation (ChIP) assay}

ChIP assay was performed using the ChIP kit (Upstate Biotechnology, USA) following the manufacturer's protocol. Briefly, SK-MEL-2 cells $\left(1 \times 10^{7}\right.$ cells for one assay) were cross-linked in $1 \%$ formaldehyde at room temperature for $10 \mathrm{~min}$ to cross-link proteins and DNAs, followed by sonication to shear the DNAs to an average size of $200-1000$ bp. Immunoprecipitation was carried out using $4 \mu \mathrm{g}$ of CREB (Cell Signaling Technology, USA), c-ETS-1 (Santa Cruz Biotechnology, USA), AP-1 (Santa Cruz Biotechnology, USA), NF- $\kappa B$ (Santa Cruz Biotechnology, USA) antibodies. After reversal of cross-linking, the DNA fragments were purified by phenol extraction and ethanol precipitation, followed by PCR analysis using primers flanking the NF- $\kappa \mathrm{B}$, c-Ets-1, AP-1 and CREB binding sites on the hST8Sia I promoter: c-ETS-1-5':ACCAATCCCCGGGCGTTT (Forward), c-ETS-1-3': GCCGCACCAAGTCCTTGGA (Reverse), CREB-5': CGTGTGTGTGTGTGTGTGTGTGTGTG (Forward), CREB-3' CCGGTGTGCCCAGGCTGT (Reverse), AP-1-5': GACTA GGGTGACGGCAGCAGG (Forward) AP-1-3': CCCCCAC CCGCAAAATTG (Reverse), NF- «B-5': CTCCGCCACACTC AGGGACT (Forward), NF- $\kappa B-3$ ': ACAAACGCCCGGGGA TTG (Reverse).

\section{Results}

\section{Effect of RA on hST8Sia I mRNA expression in SK-MEL-2 cells}

To investigate whether RA can affect hST8Sia I gene expression in SK-MEL-2 cells, cells were treated for $24 \mathrm{hr}$ with various concentration of RA. The hST8Sia I mRNA levels were analyzed by semi-quantitative RT-PCR. Treatment of cells with RA significantly decreased the levels of hST8Sia 
I mRNA expression at the concentration of $70 \mu \mathrm{M}$ (Fig. 1A). On the other hand, $\beta$-actin mRNA expression, as internal standard, was not affected by treatment of RA. Time-dependence decrease of hST8Sia I mRNA expression was detected at $24 \mathrm{hr}$ after RA treatment and decreased for periods up to $48 \mathrm{hr}$. These results clearly show that the expression of hST8Sia I was down-regulated by RA.

Analysis of transcriptional activity of hST8Sia । promoter by RA in SK-MEL-2 cells

To determine whether the 5 -flanking sequence of the hST8Sia I gene contained a RA-responsive promoter, we prepared luciferase constructs carrying serial 5 'deletions of the hST8Sia I promoter, transfected them into SK-MEL-2 cells, and then treated the transfected cells with RA. We monitored the subsequent expression of the luciferase reporter gene using the dual-luciferase reporter assay system, after

(A)

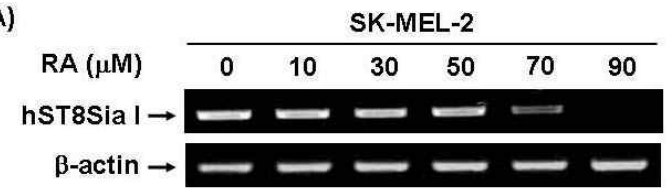

(B)
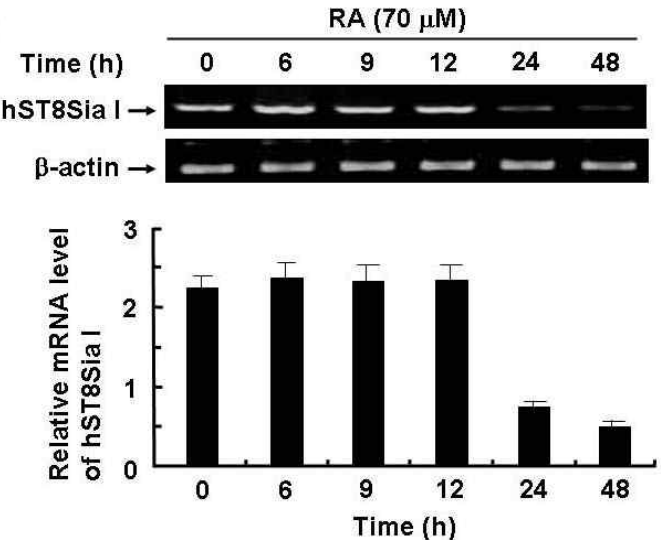

Fig. 1. RT-PCR analysis of hST8Sia I mRNA expression levels in RA-treated SK-MEL-2 cells. Total RNA from SK-MEL-2 cells was isolated after RA treatment at different concentrations $(0,10,30,50,70 \mu \mathrm{M})$ and for various times $(0,9,12,24$ or $48 \mathrm{hr})$. hST8Sia I mRNA was detected by RT-PCR. As an internal control, parallel reactions were performed to measure levels of the housekeeping gene $\beta$-actin. (A) and (B) show dose-dependent effect and time-dependent effect of RA, respectively. The densitometric intensity of hST8Sia I band was shown in under panel. Data represent the relative values \pm SD of three independent experiments and the mean values from each experiment were compared using ANOVA followed by Duncan's tests. Values not sharing the same letter are significantly different from one another $(p<0.05)$. which we measured luciferase activity with a luminometer. As shown in Fig. 2, cells harboring the pGL3-1146/-646 construct showed a remarkable decrease in luciferase activity after RA treatment, about two-fold higher than untreated transfected cells. In contrast, RA stimulation did not alter significantly the luciferase activity in cells expressing the pGL3-basic (negative control) or other $5^{\prime}$-deleted hST8Sia I promoter constructs. These results clearly suggest that the region containing nucleotides -1146 to -646 played an important role in the expression of hST8Sia I and its functions as the RA-repressive promoter in SK-MEL-2 cells.

\section{Identification of RA-responsive element in nucleotide -1146 to -646 region of hST8Sia I promoter}

Previous studies conducted in our lab demonstrated that the region from -1146 to -646 contained putative binding sites such as c-Ets-1, AP-1, CREB and NF- $\kappa$ B binding sites $[11,12]$. To determine whether these binding sites contributed to RA-suppressed expression of hST8Sia I in SK-MEL-2 cells, four mutants (pGL3-1146/-646mtCREB, mtAP-1, mtNF- $\kappa B$ and mtc-Ets-1) were used, which con-

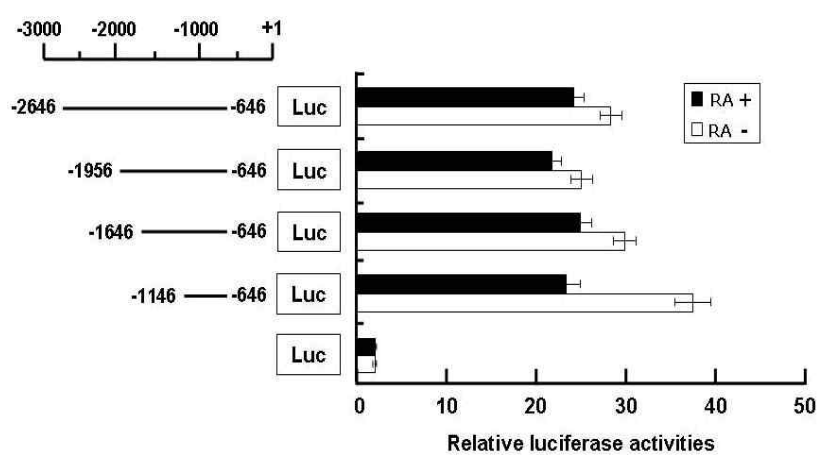

Fig. 2. Deletion analysis of hST8Sia I promoter in SK-MEL-2 cells before and after RA treatment. A schematic representation of DNA constructions containing three equal lengths from different starts of the 5 -flanking region of hST8Sia I gene linked to the luciferase reporter gene is presented. The length sizes are shown and the translation start site is indicated as +1 . pGL3-Basic without any promoter and enhancer was used as a negative control. Each construct was co-transfected into SK-MEL-2 cells with pRL-TK as an internal control. The transfected cells were incubated in the presence (solid bar) or absence (open bar) of $70 \mu \mathrm{M}$ RA for $48 \mathrm{~h}$. Relative firefly luciferase activity was measured using the Dual-Luciferase Reporter Assay System, and all firefly activity was normalized to the Renilla luciferase activity derived from pRL-TK. The values represent the means \pm SD of three independent experiments with triplicate measurements. 
tained the exact same construct as wild type pGL3-1146/-646 except that combined nucleotides within these binding sites had been changed [12]. A series of substituted mutations of luciferase constructs (Fig. 3A) were transfected into SK-MEL-2 cells and luciferase assays were carried out. The activity of each construct was compared to that of pGL3-basic and wild type (pGL3-1146/-646) as negative and positive controls, respectively. As shown in Fig. 3A, pGL3-1146/ $-646 \mathrm{mtNF}-\mathrm{kB}$ of four constructed mutations markedly reduced transcriptional activity, regardless of RA treatment, to about 6-fold of pGL3-1146/-646wt, whereas the activities of the pGL3-1146/-646mtCREB, mtAP-1 and mtc-Ets-1 con-

\section{(A)}

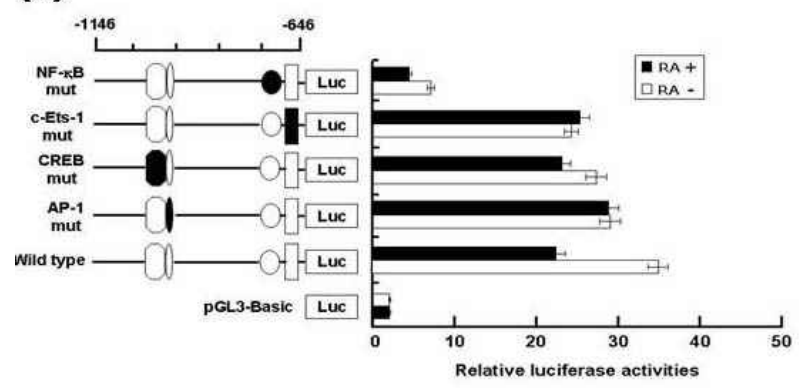

(B)

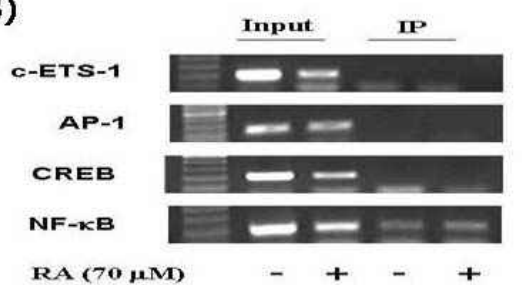

Fig. 3. Mutation promoter assay for the transcription factor binding sites in hST8Sia I gene and ChIP analysis of the hST8Sia I promoter. (A) pGL3-Basic without any promoter and enhancer was used as a negative control. Each construct was co-transfected into SK-MEL-2 cells with pRL-TK as an internal control. The transfected cells were incubated in the presence (solid bar) or absence (open bar) of $70 \mu \mathrm{M}$ RA for $48 \mathrm{hr}$. Relative firefly luciferase activity was measured using the Dual-Luciferase Reporter Assay System, and all firefly activity was normalized to the Renilla luciferase activity derived from pRL-TK. The values represent the means $\pm S D$ of three independent experiments with triplicate measurements. The mutation mark of promoter construction is indicated by closed form or opened form (wile-type). (B) PCR amplification in the -1146 and -646 region of the hST8Sia I promoter on immunoprecipitated chromatin obtained from SK-MEL-2 cells treated or not treated with RA. The input (10-fold diluted) represents the positive control. structs were not significantly changed. These results indicate that this NF-kB site is crucial for the hST8Sia I expression in SK-MEL-2 cells, and that NF- $\mathrm{kB}$ binding to this site is involved in RA-stimulated SK-MEL-2 cells. We also performed ChIP assay to confirm the binding of NF- $\mathrm{kB}$ to hST8Sia I promoter in SK-MEL-2 cells. An amplification of the hST8Sia I promoter regions was obtained in the presence of antibodies to c-ETS-1, CREB, AP-1, and NF-kB. As shown Fig. $3 B$, only NF- $k B$ has the specific amplification and DNA-protein complex observed in SK-MEL-2 cells to regulate the expression of hST8Sia I gene. There was no detectable binding in a control assay without antibody. These results support that hST8Sia I gene expression was modulated by interaction between the nuclear protein, NF- $\mathrm{kB}$ and NF- $\mathrm{k}$ B elements at nucleotide positions -731 and -722 .

Transcriptional repression of hST8Sia I via ERK pathway in SK-MEL-2 cells induced by RA

We also investigated whether RA-induced transcriptional activity of a pGL3-1146/-646-containing NF- $\kappa B$ was suppressed via PKC and JNK signal pathways. RT-PCR showed that expression of hST8Sia I mRNA was decreased in RA-treated SK-MEL-2 cells, compared to untreated SK-MEL-2 cells (Fig. 4A). Both protein kinase C (PKC) inhibitor GÖ6976 and extracellular signal-regulated protein kinase (ERK) inhibitor U0126 did not inhibit hST8Sia I expression in the RA-treated SK-MEL-2 cells. However, hST8Sia I expression in RA-treated SK-MEL-2 cells was blocked by phosphatidylinositol-3 kinase (PI-3K), p38 mitogen-activated protein kinase (MAPK), and JNK inhibitors (wortmannin, SB203580 and SP600125), respectively, as observed in RA-treated SK-MEL-2 cells in the absence of chemical inhibitors. As shown in Fig. 4B, the activity of pGL3-1146/-646 was decreased in RA-treated SK-MEL-2 cells, compared to untreated SK-MEL-2 cells. The promoter activity of pGL3-1146/-646 in SK-MEL-2 cells stimulated by RA was not significantly inhibited by PKC and ERK, compared to SK-MEL-2 cells induced by RA in absence of chemical inhibitors, as evidenced by luciferase promoter assay. However, PI-3K, JNK and p38 MAPK inhibitors resulted in a decrease of pGL3-1146/-646 activity in RA-treated SK-MEL-2 cells. These results indicated that promoter activity and mRNA transcription of the hST8Sia I gene were down-regulated by PKC and ERK signaling pathways in RA-treated SK-MEL-2 cells. 


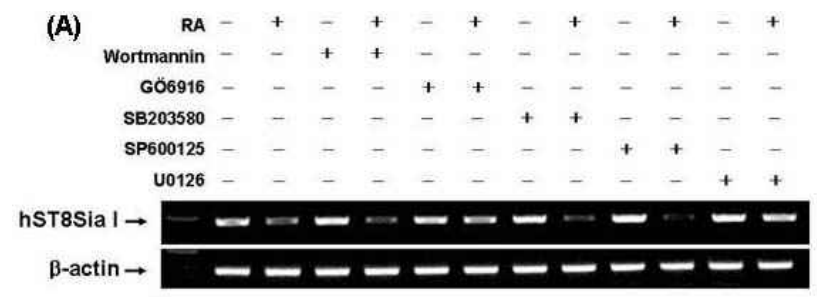

(B)

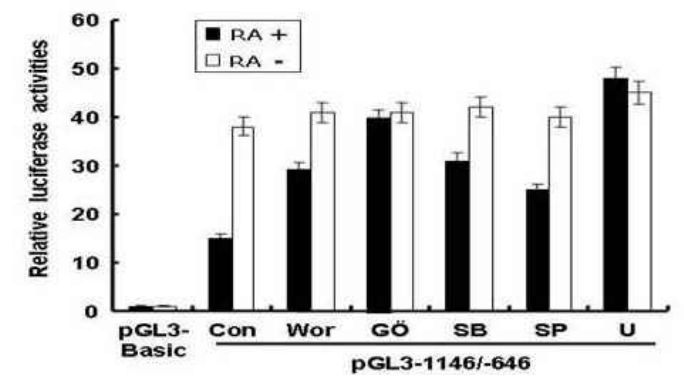

Fig. 4. Transcriptional inhibition of hST8Sia I through PKC/ ERK-signal pathways in RA-treated SK-MEL-2 cells. (A) SK-MEL-2 cells were treated with GÖ6976 $(5 \mu \mathrm{M})$, U0125 $(5 \mu \mathrm{M})$, wortmannin $(200 \mathrm{nM})$, SP600125 $(10 \mu \mathrm{M})$, and SB203580 $(20 \mu \mathrm{M})$ inhibitors in the absence or presence of RA $(70 \mu \mathrm{M})$ for $48 \mathrm{hr}$ in serum free DMEM medium, respectively. Total RNA from these cells was isolated and hST8Sia I mRNA was detected by RT-PCR analysis. Beta-actin was included as an internal control. (B) The pGL3-1146/-646 was cotransfected into SK-MEL-2 cells with pRL-TK as the internal control. The transfected cells were incubated in the presence and absence of 70 $\mu \mathrm{M}$ RA with each inhibitor for $48 \mathrm{hr}$. All firefly activity was normalized to the Renilla luciferase activity derived from $\mathrm{pRL}-\mathrm{TK}$. The values represent the mean $\pm \mathrm{SD}$ for three independent experiments with triplicate measurements.

\section{Discussion}

Previous studies have shown that human melanoma cells have high levels of ganglioside GD3 and hST8Sia I activity, and mRNA levels for the enzyme are coincidently high $[4,17,18]$. We have also demonstrated that the expression level of hST8Sia I gene is specifically high in human melanoma cell line SK-MEL-2 [11]. In this study, we demonstrated for the first time that RA down-regulated the expression of hST8Sia I mRNA in human melanoma cells. Moreover, this decrease was time-dependent. The decreased hST8Sia I mRNA signal was detected after $24 \mathrm{hr}$ of RA treatment, and then this signal decreased up to the 48 -h time point. We also revealed that the promoter region of the hST8Sia I gene contained RA-responsive element. Previous studies have shown that RA inhibits the proliferation and induces apoptosis of melanoma cells in vitro $[6,15]$. In line with these re- ports, we observed that RA inhibited the proliferation of SK-MEL-2 cells, and that it induced apoptosis after a 48-h treatment of the cells with $70 \mu \mathrm{M}$ RA.

We also unraveled a part of the transcriptional regulation mechanism that underlies hST8Sia I gene repression in response to RA signaling. In order to investigate RA-responsive elements involved in the suppressed expression of the hST8Sia I gene in SK-MEL-2 cells, we first sought to identify the region within the hST8Sia I promoter that was critical for RA-repressed gene expression. We isolated the region between -1146 and -646 as the core promoter; this region was required for transcriptional repression of hST8Sia I in RA-treated SK-MEL-2 cells. Previous reports from our lab revealed several transcription factor binding sites such as c-ETS-1, AP-1, CREB and NF- $\kappa$ B binding sites in this region $[11,12]$. We have also demonstrated that only the NF- $\kappa B$ binding site at position at -731 to -722 in this region contributes to promoter activity necessary for high expression of hST8Sia I in Fas-induced Jurkat T cells [12] and human melanoma SK-MEL-2 cells [11]. Contrary to these findings, however, our present site-directed mutagenesis and ChIP analyses indicated that binding to this NF- $\kappa B$ element mediated RA-dependent down-regulation of hST8Sia I gene expression in SK-MEL-2 cells.

$\mathrm{NF}-\kappa \mathrm{B}$ is a crucial transcription factor that controls the expression of various genes involved in immune and inflammatory responses, cell cycle progression, apoptosis, and oncogenesis [1,2]. Although a synthetic RA (CD437) induces apoptosis of human melanoma A375 cells through NF- $\kappa B$ activation by RIG pathway [15], down-regulation of NF- $\kappa$ B-mediated gene expression by RA stimulation in human melanoma cells have not been reported. Therefore, it is important to elucidate which signaling pathways are upstream of this NF- $\kappa B$-mediated repression of the hST8Sia I gene expression. In the present study, our data showed that PKC and ERK were blocked by RA in SK-MEL-2 cells. The transcriptional repression of hST8Sia I was associated with PKC/ERK pathways induced by RA in SK-MEL-2 cells, which has not been reported yet. PKC and ERK inhibitors did not inhibited the expression of hST8Sia I, but PI-3K, PKC, p38 MAPK, and JNK inhibitors decreased the expression levels of hST8Sia I in SK-MEL-2 cells stimulated by RA. These results suggest that RA-mediated transcriptional repression of hST8Sia I may be related to PKC/ERKdependent pathways, as illustrated in Fig. 5.

Although the precise mechanisms involved in the 


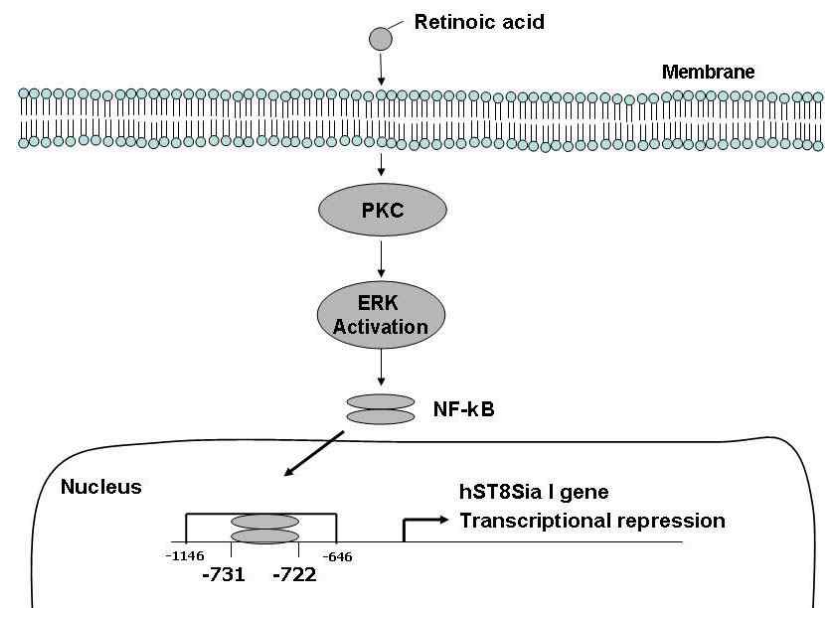

Fig. 5. Schematic diagram illustrating transcriptional regulation of hST8Sia I gene expression in RA-induced SK-MEL-2 cells.

RA-mediated activation of NF- $\kappa \mathrm{B}$ leading to a transcriptional down-regulation of the hST8Sia I gene are unknown, we have demonstrated here for the first time that the PKC/ERK-dependent NF- $\kappa B$ activation down-regulates the expression of hST8Sia I in RA-stimulated SK-MEL-2 cells.

\section{Acknowledgment}

This study was supported by research funds from Dong-A University.

\section{References}

1. Baeuerle, P. A. and D. Baltimore. 1996. NF-kB: ten years after. Cell 87, 13-20.

2. Chen, F., V. Castranova, and X. Shi. 2001. New insights into the role of nuclear factor- $\mathrm{kB}$ in cell growth regulation. $A m$ J. Pathol. 159, 387-397.

3. Cheung, N. K., U. M. Saarinen, J. E. Neely, B. Landmeier, D. Donovan, and P. F. Coccia. 1985. Monoclonal antibodies to a glycolipid antigen on human neuroblastoma cells. Cancer Res. 45, 2642-2649.

4. Dippold, W. G., K. O. Lloyd, L. T. Li, H. Ikeda, H. F. Oettgen, and L. J. Old. 1980. Cell surface antigens of human malignant melanoma: definition of six antigenic systems with mouse monoclonal antibodies. Proc. Natl. Acad Sci. USA, 77, 6114-6118.

5. Fields, A. L., D. R. Soprano, and K. J. Soprano. 2007. Retinoids in biological control and cancer. J. Cell Biochem 102, 886-898.

6. Fligiel, S. E., D. R. Inman, H. S. Talwar, G. J. Fisher, J. J. Voorhees, and J. Varani. 1992. Modulation of growth in normal and malignant melanocytic cells by all-trans retinoic acid. J. Cutan Pathol. 19, 27-33.

7. Fukuda, M., K. Horibe, and K. Furukawa. 1998. Enhancement of in vitro and in vivo anti-tumor activity of anti-GD2 monoclonal antibody 220-51 against human neuroblastoma by granulocyte-macrophage colony-stimulating factor and granulocyte colony-stimulating factor. Int. J. Mol. Med 2, 471-475.

8. Hakomori, S. 1996. Tumor malignancy defined by aberrant glycosylation and sphingo (glyco) lipid metabolism. Cancer Res. 56, 5309-5318.

9. Hakomori, S. 1981.lycosphingolipids in cellular interaction, differentiation, and oncogenesis. Annu. Rev. Biochem 50, 733-764.

10. Haraguchi, M., S. Yamashiro, A. Yamamoto, K. Furukawa, K. Takamiya, K. O. Lloyd, H. Shiku, and K. Furukawa. 1994. Isolation of GD3 synthase gene by expression cloning of GM3 a-2,8-sialyltransferase cDNA using anti-GD2 monoclonal antibody. Proc. Natl. Acad Sci. USA 91, 10455-10459.

11. Kang, N. Y., C. H. Kim, K. S. Kim, J. H. Ko, J. H. Lee, Y. K. Jeong, and Y. C. Lee. 2007. Expression of the human CMP-NeuAc:GM3 a2,8 sialyltransferase (GD3 synthase) gene through the NF- $\mathrm{kB}$ activation in human melanoma SK-MEL-2 cells. Biochim Biophys. Acta 1769, 622-630.

12. Kang, N. Y., S. K Kang, Y. C. Lee, H. J. Choi, Y. S. Lee, S. Y. Cho, Y. S. Kim, J. H. Ko, and C. H. Kim. 2006. Transcriptional regulation of the human GD3 synthase gene expression in Fas-induced Jurkat $\mathrm{T}$ cells: a critical role of transcription factor $\mathrm{NF}-\mathrm{\kappa B}$ in regulated expression. Glycobiology 16, 375-389.

13. Merritt, W. D., J. T. Casper, S. J. Lauer and G. H. Reaman. 1987. Expression of GD3 ganglioside in childhood T-cell lymphoblastic malignancies. Cancer Res. 47, 1724-1730.

14. Old, L. J. 1981. Cancer immunology: the search for specificity--G. H. A. Clowes Memorial lecture. Cancer Res. 41, 361-375.

15. Pan, M., S. Geng, S. Xiao, J. Ren, Y. Liu, X. Li, Z. Li, and Z. Peng. 2009. Apoptosis induced by synthetic retinoic acid CD437 on human melanoma A375 cells involves RIG-I pathway. Arch Dermatol. Res. 301, 15-20.

16. Portoukalian, J., G. Zwingelstein, and J. F. Dore. 1979. Lipid composition of human malignant melanoma tumors at various levels of malignant growth. Eur. J. Biochem 94, 19-23.

17. Pukel, C. S., K. O. Lloyd, L. R. Travassos, W. G. Dippold, H. F. Oettgen, and L. J. Old. 1982. GD3, a prominent ganglioside of human melanoma. Detection and characterisation by mouse monoclonal antibody. J. Exp. Med 155, 1133-1147.

18. Rusan, S., B. K. Raj, and K. O. Lloyd. 1999. Relationship of glycosyltransferases and mRNA levels to ganglioside expression in neuroblastoma and melanoma cells. J. Neurochem 72, 514-521.

19. Sasaki, K., K. Kurata, N. Kojima, N. Kurosawa, S. Ohta, N. Hanai, S. Tsuji, and T. Nishi. 1994. Expression cloning of a GM3-specific a-2,8-sialyltransferase (GD3 synthase). J. Biol. Chem 269, 15950-15956.

20. Sporn, M. B. and A. B. Roberts. 1983. Role of retinoids in 
differentiation and carcinogenesis, Cancer Res. 43, 3034-3040.

21. Svennerholm, L. 1980. Gangliosides and synaptic transmission. Adv. Exp. Med Biol. 125, 533-544.

22. Thampoe, I. J., K. Furukawa, E. Vellvé, and K. O. Lloyd. 1989. Sialyltransferase levels and ganglioside expression in melanoma and other cultured human cancer cells. Cancer Res. 49, 6258-6264.

23. Welte, K., G. Miller, P. B. Chapman, H. Yuasa, E. Natoli, J. E. Kunicka, C. Cordon-Cardo, C. Buhrer, L. J. Old, and A. N. Houghton. 1987. Stimulation of T lymphocyte proliferation by monoclonal antibodies against GD3 ganglioside. J. Immunol. 139, 1763-1771.

24. Yamaguchi, H., K. Furukawa, S. R. Fortunato, P. O.
Livingston, K. O. Lloyd, H. F. Oettgen, and L. J. Old. 1987. Cell-surface antigens of melanoma recognized by human monoclonal antibodies. Proc. Natl. Acad Sci. USA 84, 2416-2420.

25. Yamashiro, S., M. Okada, M. Haraguchi, K. Furukawa, K. O. Lloyd, H. Shiku, and K. Furukawa. 1995. Expression of a2,8-sialyltransferase (GD3 synthase) gene in human cancer cell lines: high level expression in melanomas and up-regulation in activated T lymphocytes. Glycoconj. J. 12, 894-900.

26. Zhang, H., S. Zhang, N.-K. V. Cheung, G. Ragupathi, and P. O. Livingston. 1998. Antibodies against GD2 ganglioside can eradicate syngeneic cancer micrometastases. Cancer Res. 58, 2844-2849.

\section{초록 : 흑색종세포주 SK-MEL-2에서 레티노이드에 의한 GD3합성효소(hST8Sia I)의 발현억제 \\ 권화영 · 강남영 · 이영춘* \\ (동아대학교 생명자원과학대학 생명공학과)}

흑색종세포주 SK-MEL-2에서 레티노이드에 의한 GD3합성효소(hST8Sia I)의 발현억제기작을 규명하게 위하여 hST8Sia I의 프로모터 활성을 조사해 본 결과 -1146에서 -646영역에서 레티노이드에 의한 활성억제를 나타내었 다. 또한 부위특이적 변이와 ChIP분석은 -731에서 -722영역에 위치한 전사인자NF-kB 결합부위가 hST8Sia I의 레 티노이드에 의한 활성억제에 중요하게 관여하고 있음을 나타내었다. 이러한 발현 억제는 PKC/ERK 신호전달경 로를 통하여 일어난다는 것을 신호전달경로 저해제를 이용한 RT-PCR과 프로모터 활성조사에 의해 규명하였다. 